\title{
An Investigation of the Longitudinal Dynamic Stability of a Box-Wing Aircraft
}

\author{
Paul Olugbeji Jemitola, Paul Peter Okonkwo* \\ Email address: \\ pojemitola@yahoo.co.uk (P. O. Jemitola), pokonkwol@gmail.com (P. P. Okonkwo) \\ ${ }^{*}$ Corresponding author
}

Department of Aerospace Engineering, Faculty of Air Engineering, Air Force Institute of Technology, Kaduna, Nigeria

\section{To cite this article:}

Paul Olugbeji Jemitola, Paul Peter Okonkwo. An Investigation of the Longitudinal Dynamic Stability of a Box-Wing Aircraft. International Journal of Mechanical Engineering and Applications. Vol. 9, No. 5, 2021, pp. 79-84. doi: 10.11648/j.ijmea.20210905.12

Received: October 19, 2021; Accepted: November 15, 2021; Published: November 27, 2021

\begin{abstract}
This study was undertaken to identify the longitudinal dynamic characteristics of the Box-Wing Aircraft. Research on the Box Wing aircraft gained traction following increasing demand for an environmental friendly aircraft that would reduce noise and pollutant emissions. The Box-Wing Aircraft is renowned for its potential aerodynamics advantages over conventional aircraft. However, due to its departure from conventional configuration, the stability dynamics of the Box Wing has not been fully explored. This study investigates the longitudinal dynamic stability characteristics of a Box Wing Aircraft at the conceptual design level using the J2 Aircraft Dynamics Software. The study integrates empirical mass estimation methods and aerodynamic data generated from a vortex lattice tool into J2 Aircraft Dynamics Software Suite. This was done to assess the Short Period Oscillation and Phugoid responses of a Box Wing Aircraft and a conventional cantilever wing aircraft. For a 2s step input, the short period oscillation induced on the Box Wing was damped out after 19s resulting in damping ratio of 0.68 and the undamped natural frequency is $1.8 \mathrm{rad} / \mathrm{s}$. In the Phugoid mode, the Box Wing Aircraft gives a damping ratio of 0.006 and undamped natural frequency of $0.07 \mathrm{rad} / \mathrm{s}$. On the other hand, the SPO induced on the reference conventional aircraft settles after 14s producing a damping ratio of 0.76 and undamped natural frequency of $3.12 \mathrm{rad} / \mathrm{s}$. The damping ratio and undamped natural frequency for the Phugoid mode of the reference conventional aircraft were however 0.001 and $0.07 \mathrm{rad} / \mathrm{s}$ respectively. These results indicate that the reference conventional cantilever wing aircraft possesses acceptable longitudinal dynamic stability characteristics while the Box Wing Aircraft would require stability augmentation systems to improve its flying and handling qualities.
\end{abstract}

Keywords: Longitudinal, Dynamics, Stability, Box-Wing

\section{Introduction}

The study of the longitudinal dynamic stability of an aircraft is essential to understanding the aircraft flying and handling qualities. Unlike Box Wing aircraft, the stability and control dynamics of a conventional cantilever wing aircraft are generally well explored in literature. It was therefore necessary to investigate how the longitudinal dynamic stability of a conventional cantilever aircraft compares with the Box Wing Aircraft. Thus, following the procedure outlined in Figure 1, the dynamic stability of an optimized box wing aircraft was examined.

The study was validated using a Conventional Cantilever Wing aircraft. Aerodynamic data and mass statements were generated from an optimized reference aircraft. Subsequently, mass and c.g situations were used to generate aircraft inertia statements. The J2 [1] Aircraft Dynamics Software Suite was then employed to integrate disciplinary models and perform dynamic simulations. The longitudinal dynamic stability modes investigated included the Short Period Oscillation and Phugoid modes. These modes were investigated for a Box Wing aircraft and compared with a conventional cantilever wing aircraft.

\section{Description of the Reference Aircraft}

\subsection{Box Wing Aircraft}

This work is derived from the conceptual design study of a medium-range Box Wing Aircraft conducted in Cranfield 
University and out- lined in a PhD Thesis by Jemitola [2]. The reference Box Wing aircraft (see Figure 2) is a $4000 \mathrm{~nm}$ range airliner with a maximum take-off mass of $127760 \mathrm{~kg}$ and wingspan of $37.6 \mathrm{~m}$. The fore and aft wing gross areas are $118.32 \mathrm{~m}^{2}$ each. The wing gap, measured at the wing tips, is $8.0 \mathrm{~m}$ while the fore and aft wing sweep angles are 40 and -25 degrees respectively. Overall fuselage length is $46 \mathrm{~m}$ and maximum diameter is $5.6 \mathrm{~m}$.

\subsection{Conventional Cantilever Wing Aircraft}

To compare and validate applied methodology, a conventional cantilever wing aircraft similar to the B-767 Aircraft, and obtained from Jemitola [2] was studied, see Figure 3. The selected cantilever aircraft is also a $4000 \mathrm{~nm}$ range airliner but with a maximum take- off mass of 136000 $\mathrm{kg}$ and wingspan of $47.0 \mathrm{~m}$. The wing gross area is equivalent to the sum of the fore and aft wing areas of the baseline Box Wing aircraft at $236.64 \mathrm{~m}^{2}$, while the wing sweep angle is 30 degrees. Overall fuselage length is $46 \mathrm{~m}$ and maximum diameter is $5.6 \mathrm{~m}$.

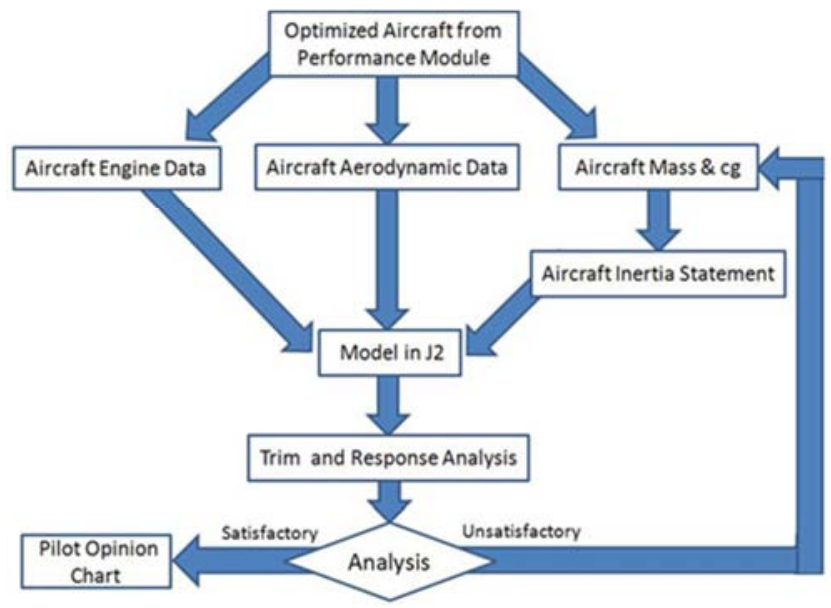

Figure 1. Stability and Control Evaluation Schematic.

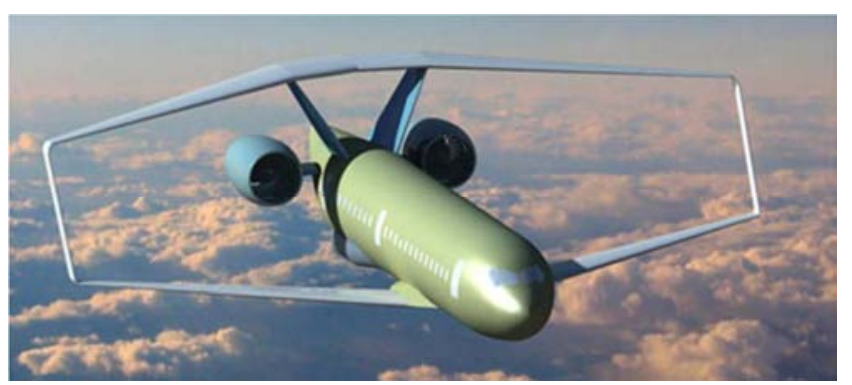

Figure 2. Box Wing Aircraft.

\section{Aircraft Mass and Inertia Statements}

The flight dynamic behaviour of an aircraft is influenced by its mass and distribution. It was therefore imperative to determine the aircraft component masses as well as their inertia.

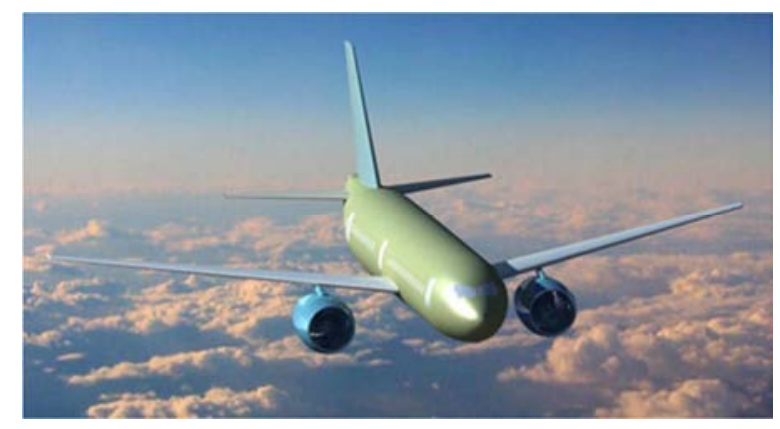

Figure 3. Conventional Aircraft.

\subsection{Mass}

To produce the mass statements used in this study, the masses of the fuselage, nacelle, propulsion, landing gear, surface controls and fixed equipment of both the conventional Cantilever Wing and the Box Wing aircraft were estimated using the methods given in Jenkinson [3]. The fore and aft wing, tip fin and tail fin masses of the Box Wing aircraft were computed using the algorithm given in Jemitola [4]. Mission fuel for both aircraft were obtained from the Breguet Range Equation as defined by Matthews [6] and presented in Equation 1.

$$
R=\frac{L}{D}=\frac{(\text { Cruisespeed })}{s f c} \ln \frac{\left(W_{0}\right)}{W_{1}}
$$

Where:

$W_{o}=$ Initial aircraft mass

$W_{l}=$ Final aircraft mass

Wing fuel volume to accommodate the mission fuel was estimated using Jenkinson method [5]. Finally, the $O E M$ and $M_{\text {TOM }}$ mass statements were produced.

\subsection{Inertia}

The outcomes of the preceding section were used to develop the aircraft inertia statements. Following Bruhn [7] inertia estimation concept, the inertia of each component was first determined about its own centroidal axis and then about the axes of the aircraft. Subsequently, the inertia mass statements for both aircraft were produced.

\section{Aircraft Aerodynamic and Engine Data}

As a prelude to the longitudinal dynamic stability analysis using J2 Aircraft Dynamics Software Suite, it was necessary to generate relevant aerodynamic data with which the software would perform the analysis. The data required to accomplish this objective include the following:

1. Variation of the fore wing lift coefficient with angle of attack and elevon deflection.

2. Variation of aft wing lift curve slope with angle of attack and elevator deflection.

3. Variation of fore wing trim drag as a function of angle of attack and elevon deflection.

4. Variation of the aft wing trim drag as a function of angle 
of attack and elevator deflection.

5. Aircraft pitching moment as a function of aft wing angle of attack and elevator and elevon deflection.

6. Engine thrust as a function of Mach number, altitude and engine throttle setting.

Serials 1 to 5 above were initially computed using Roskam Methods [8], ESDU74011 [9] and ESDU89029 [10] sheets. However, due to the complexity and volume of computations required, Javafoil [11] was subsequently used after the preliminary set of computations. Javafoil [11] is a soft- ware applies potential flow and boundary layer analysis to determine aerodynamic characteristics and stability derivatives for airfoils and aircraft models. The results from Javafoil [11] were verified with hand calculations.

Engine thrust as a function of Mach Number, altitude and engine throttle setting was computed using methods given by Yechout et al [12]. These calculations were derived for both the Box Wing and Conventional Cantilever Wing aircraft. Values obtained were then used to populate the aircraft models in J2 Aircraft Dynamics Software Suite.

\section{Longitudinal Dynamics}

The longitudinal dynamics of an aircraft may be likened to a pair of loosely coupled mass-spring-damper systems. The interpretation of the motion of the aeroplane following a disturbance from equilibrium may be compared to the behaviour of the mechanical mass-spring-damper. However, unlike the mass-spring damper system, the damping and frequency characteristics of the aircraft are not mechanical but dependent on its aerodynamic characteristics [13].

\subsection{Short Period Oscillation}

Short Period Oscillation (SPO) mode is typically a damped oscillation in pitch about the lateral axis. The principal variables involved in SPO are the angle of attack, pitch rate and pitch attitude.

SPO may be initiated by applying a short duration disturbance in pitch to the trimmed aircraft. This is achieved with an elevator pulse sufficiently short so as not to excite the Phugoid mode. The SPO evaluations were then located into a longitudinal short period pilot opinion contours chart, otherwise called the 'Thumbprint' criterion shown in Figure 4. The 'Thumbprint' criterion guides aircraft designers and evaluators on the best combinations of longitudinal short period mode damping and frequency that would guarantee good handling qualities. The chart is empirical and is based entirely on pilot's opinion. It is however considered adequate for conceptual level design studies.

The 'Thumbprint' depicts the plots of undamped natural frequency against damping ratio on a plain marked as satisfactory, acceptable, poor and unacceptable. Consequently, by locating the un- damped natural frequencies and damping ratios of a model on a 'Thumbprint', a fair assessment of the acceptability of aircraft's SPO response can be made for conceptual design level studies.

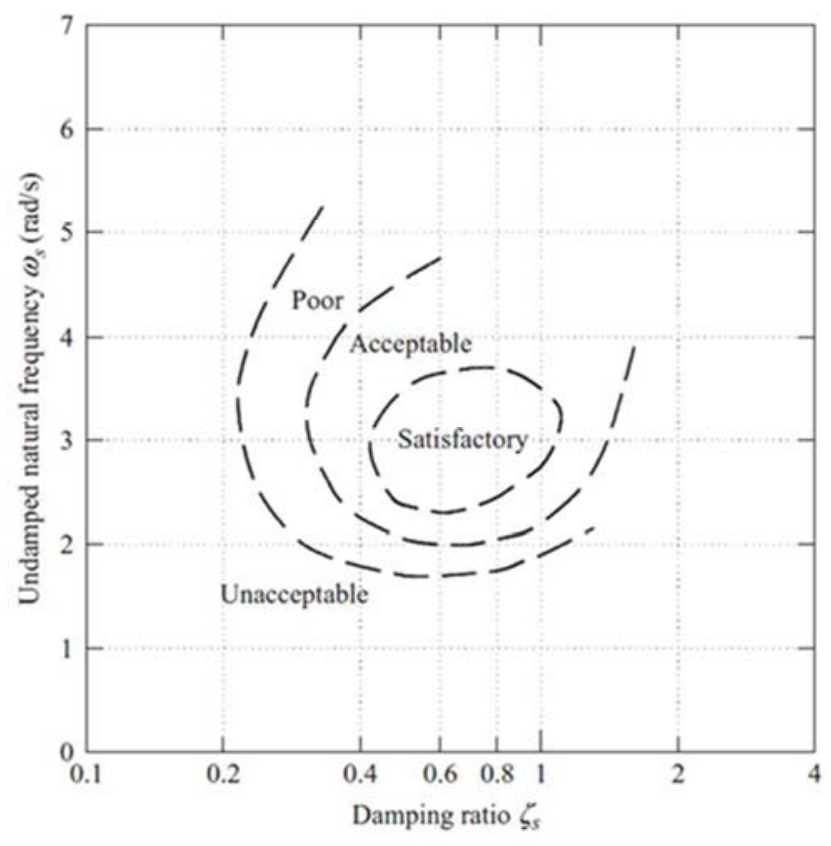

Figure 4. Longitudinal Short Period Pilot Opinion Contours [13].

\subsubsection{Short Period Oscillation - Conventional Cantilever \\ Wing Aircraft}

The model of the conventional cantilever wing aircraft for an arbitrarily chosen $33 \%$ payload was simulated for flight at an altitude of $31,000 \mathrm{ft}$ and Mach 0.8 . After a period of $2 \mathrm{~s}$, a step input of $0.02 \mathrm{~s}$ was introduced by deflecting the model's elevator. Figure 5 shows the behaviour of the aircraft. The $y$-axis represents the aircraft's angle of attack in degrees and the $\mathrm{x}$-axis shows the time in seconds. When perturbed, there was a sudden decrease in angle of attack followed by an overshoot above the trim angle of attack, then the oscillation damps out. The overall change in angle of attack during the oscillation is less than a degree and it settles about 14 seconds after the initial perturbation. The computed damping ratio is 0.76 and the undamped natural frequency is 3.12 $\mathrm{rad} / \mathrm{s}$.

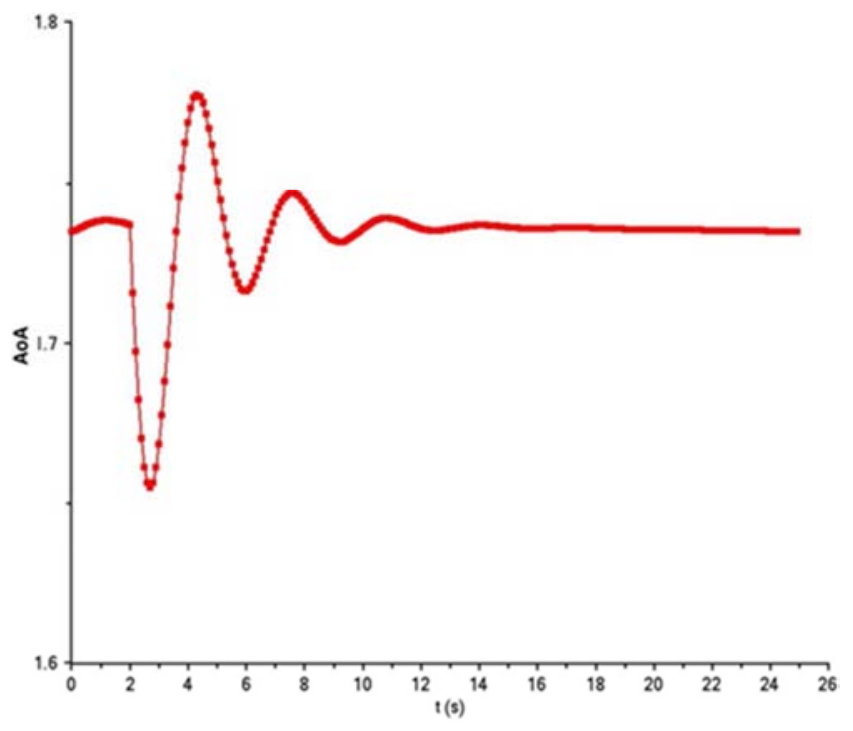

Figure 5. Short Period Oscillation - Conventional Aircraft. 


\subsubsection{Short Period Oscillation - Box Wing Aircraft}

The model of the Box Wing Aircraft also with an arbitrarily chosen $33 \%$ payload was also simulated for flight at an altitude of 31,000 ft cruising at Mach 0.8 . After a period of $2 \mathrm{~s}$, a step input of $0.02 \mathrm{~s}$ was introduced by deflecting the model's elevon and elevator in opposite directions. The behaviour of the aircraft is shown in Figure 6 where the y-axis is the aircraft's angle of attack in degrees and the $\mathrm{x}$-axis the time in seconds. At the perturbation, there is a much deeper drop in angle of attack compared to the Conventional Cantilever Wing aircraft and the reversal is shallower as the oscillation dampens out. The overall change in angle of attack during the oscillation is about 2.2 degrees and it settled 19 seconds after the initial disturbance. This gives a damping ratio of 0.68 and an undamped natural frequency of $1.8 \mathrm{rad} / \mathrm{s}$. The damping ratio of 0.68 obtained in this study is of the same order of magnitude as that derived by Scholz and Caja [14] in their investigation of the dynamics of Box wing aircraft in conceptual design stage. Scholz and Caja [14] observed that Box-Wing Aircraft possess a level 1 handling quality characteristic with a SPO damping ratio of 0.318 when manual calculation is employed and a level 2 handling quality characteristic with a damping ratio of 0.267 in SPO when the Simulation and Dynamic Stability Analyser Software is utilised.

\subsection{Phugoid}

The Phugoid is a damped harmonic motion resulting in an aircraft flying a gentle sinusoidal flight path about the nominal trimmed height datum. As large inertia and momentum effects are involved, the motion is slow. The Phugoid mode may be excited by applying a small speed disturbance to the aircraft in trimmed flight. This is best achieved by applying a small step input to the elevator which will cause the aircraft to fly up, or down, according to the sign of the input [13].

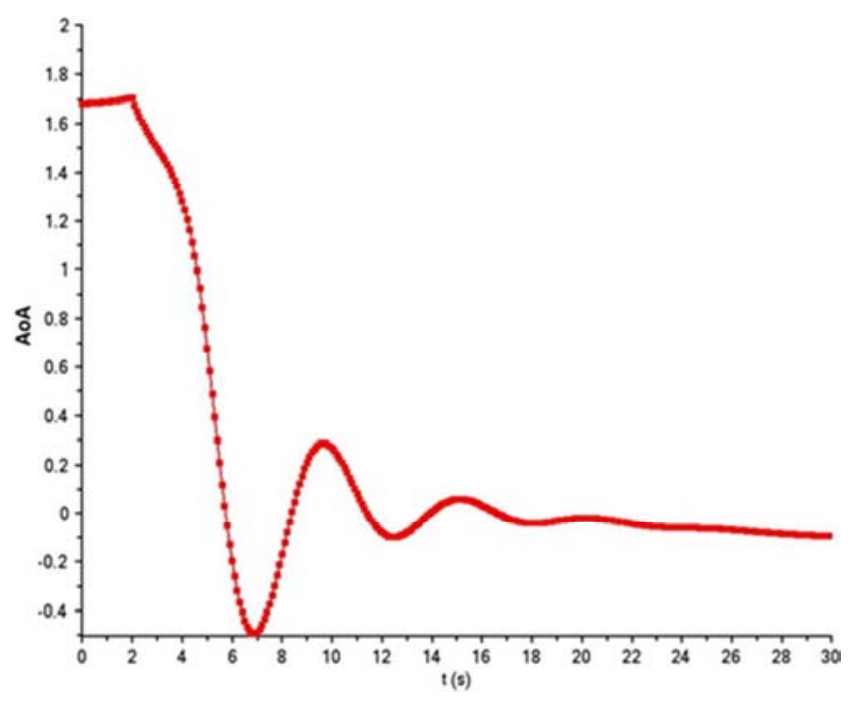

Figure 6. Short Period Oscillation - Box Wing Aircraft.

\subsubsection{Phugoid - Conventional Cantilever Wing Aircraft}

The model of the conventional cantilever wing aircraft with
$33 \%$ payload was investigated for its response to Phugoid motion at an altitude of $31,000 \mathrm{ft}$ while flying at Mach 0.8 . The behaviour of the aircraft is shown in Figure 7. The left $y$-axis represents altitude in feet, the right $y$-axis shows the true air speed while the $\mathrm{x}$-axis shows time in seconds. The trend observed is consistent with a typical Phugoid motion with the airspeed and altitude oscillating in opposition. At the end of the $250 \mathrm{~s}$ period, the speed of the aircraft was decreased by $58 \mathrm{kts}$. The computed damping ratio of 0.001 and undamped natural frequency of $0.07 \mathrm{rad} / \mathrm{s}$ are all low and typical of Phugoid motion for a conventional aircraft [13].

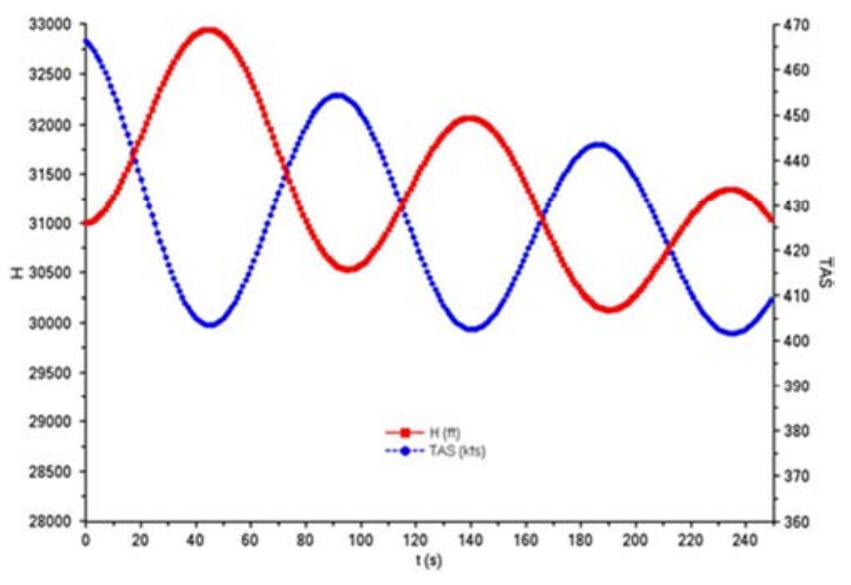

Figure 7. Conventional Aircraft Phugoid.

\subsubsection{Phugoid - Box Wing Aircraft}

The model of the Box Wing Aircraft with 33\% payload was simulated for flight at $31,000 \mathrm{ft}$ at Mach 0.8 to ascertain its response to Phugoid motion. The behaviour of the aircraft is shown in Figure 8. The left y-axis shows altitude in feet, right $\mathrm{y}$-axis shows the true air speed while the $\mathrm{x}$-axis shows the time in seconds. Similarly, the trend shown is consistent with a Phugoid with the airspeed and altitude oscillating in opposition. During the 250s period, the speed of the aircraft decreased by $56 \mathrm{kts}$. These losses are indicative of the gradual damping of the motion. The computed damping ratio of 0.006 and undamped natural frequency of $0.07 \mathrm{rad} / \mathrm{s}$ are all too low. This indicates that the aircraft is lowly damped and would require stability augmentation device to enable adequate dynamic response [15].

\section{Discussion}

The damping ratio and undamped natural frequency of the SPO response for Box Wing and conventional cantilever wing aircraft were imposed on the 'Thumbprint' criterion plot shown in Figure 9. The figure illustrates the significant difference between the longitudinal dynamic response of a conventional and the Box Wing aircraft. While the conventional aircraft falls in the satisfactory area of the 'Thumbprint' plot, the Box Wing aircraft is in the unacceptable area. This is partly due to the low undamped natural frequency $(1.8 \mathrm{rad} / \mathrm{s})$ of the Box Wing and the rather large wing area of the aft wing. If the static margin were increased to achieve satisfactory 'Thumbprint' criterion, the Box Wing Aircraft would depart further from its ideal 
arrangement for maximum aerodynamic efficiency (equal lift generation on both fore and aft wings) thus negating the primary benefit of the design. It could thus be inferred that the low undamped natural frequency in SPO enables marginal longitudinal dynamic stability; hence the need for a stability augmentation device to ensure acceptable longitudinal dynamic response for the Box Wing aircraft configuration. This would ensure that the aerodynamic efficiency of the Box-Wing concept is retained while enabling the achievement of satisfactory 'Thumbprint' criterion for acceptable longitudinal dynamic stability. Consequently, the assessment of the effects of stability augmentation system on a Box-Wing Aircraft is considered an important topic for future research in the quest for the development of an environmentally friendly airliner.

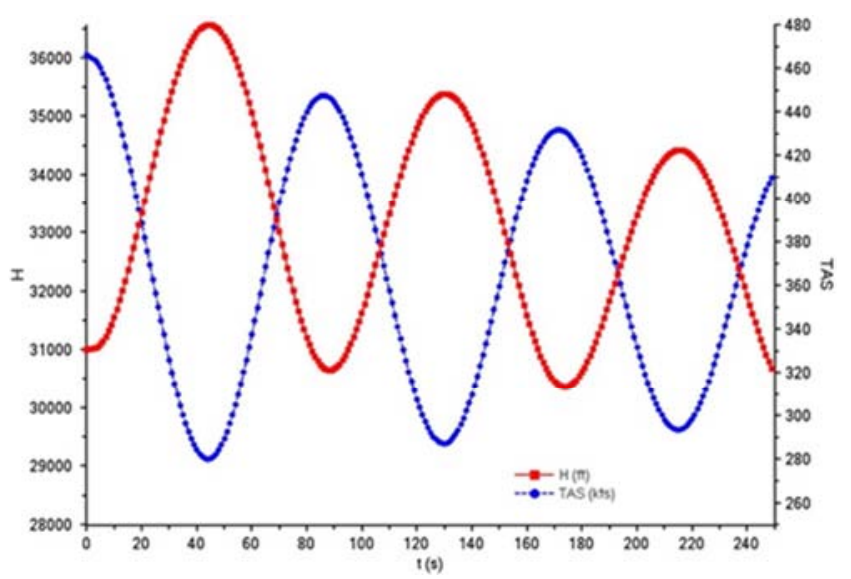

Figure 8. Box Wing Phugoid.

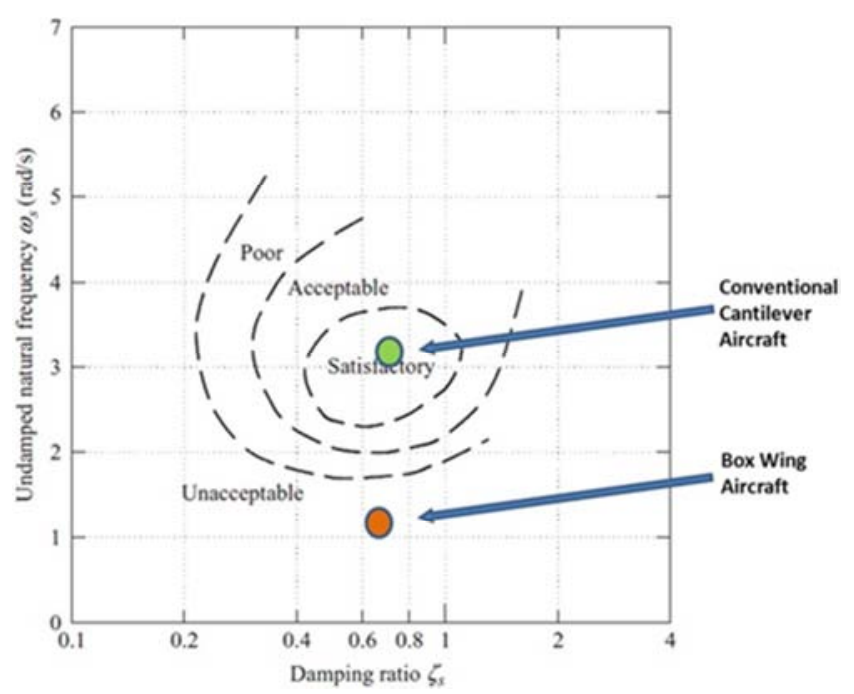

Figure 9. Thumbprint Criterion - Box Wing and Conventional Aircraft.

For the Phugoid mode, the amplitude of the Box Wing Aircraft's altitude oscillation was about 1.2 times that of the conventional aircraft. Figure 8 shows that even though the Box Wing Aircraft descended $323 \mathrm{ft}$ below start altitude compared to the conventional aircraft which climbed $724 \mathrm{ft}$ above start altitude (Figure 7), the Box Wing had oscillated much more in amplitude. A similar trend was observed when speed losses are compared in Figures 7 and 8. While the conventional aircraft lost $62 \mathrm{kts}$ in the first oscillation, the Box Wing Aircraft lost about 3 times that value. This rather high amplitude of the Box Wing oscillations, which would be sensed by any passengers in the aircraft, could be attributed to its marginal dynamic stability. Again, this further reinforces the need for stability augmentation devices on this aircraft configuration.

\section{Conclusion}

This study compared the longitudinal dynamic stability of a Box Wing Aircraft to a conventional cantilever wing configuration to determine its handling and flying quality. The study integrated mass and inertia statements as well as engine and aerodynamic data into the J2 Aircraft Dynamics Software Suite to assess the Short Period Oscillation and Phugoid responses of the Box Wing Aircraft. The results indicate that the Box Wing Aircraft would require stability augmentation devices to enhance its handling characteristics.

\section{Acknowledgements}

This paper is derived from a $\mathrm{PhD}$ Thesis by one of the authors titled 'Conceptual Design and Optimization Methodology for Box Wing Aircraft'. Accordingly, the authors acknowledge the support of the Department of Aerospace Engineering, Cranfield University and the Nigerian Air Force.

\section{References}

[1] J. Jeffery: "J2 Universal Aircraft Dynamics Software Suite.” J2 Aircraft Dynamics Ltd, (2010).

[2] P. O Jemitola: Conceptual Design and Optimisation Methodology for Box Wing Aircraft, School of Aerospace Engineering, Department of Aerospace Engineering, Cranfield University, Cranfield, (2012).

[3] L. Jenkinson, D. Rhodes and P. Simpkin: Civil Jet Aircraft Design. Arnold Publishers, UK, (1999).

[4] P. O. Jemitola, J. Fielding and G. Monterzino: 'Wing Mass Estimation Algorithm for Medium Range Box Wing Aircraft', Aeronautical Journal, (2013).

[5] L. Jenkinson and J. F. Marchman: Aircraft Design Projects for Engineering Students, Oxford, UK: Elsevier Butterworth-Heinemann and AIAA, (2003).

[6] C. Matthews: Aeronautical Engineers Data Book, Oxford, UK: Elsevier Butterworth Heinemann, (2002).

[7] E. Bruhn: Analysis and Design of Flight Vehicle Structures. Indianapolis, USA: SR Jacobs and Associates, (1973).

[8] J. Roskam: Airplane Design: Part VI - Preliminary Calculation of Aerodynamic, Thrust and Power Characteristics. Roskam Aviation and Engineering Corporation, Kansas, USA, (1990).

[9] ESDU: "Rate of change of lift coefficient with control deflection for full-span plain controls," Tech. Rep. 74011, The Royal Aeronautical Society, March (1974). 
[10] ESDU: "Installed tailplane lift-curve slope at subsonic speeds," Tech. Rep. 89029, The Royal Aeronautical Society, October (1989).

[11] M. Hepperle: "Javafoil version 2.20 - 01." http://www.mhaerotools.de/airfoils/javafoil.htm, December 2011. Accessed March (2010).

[12] T. R. Yechout, T. D. Bossert and S. Morris: Introduction to aircraft flight mechanics: perfor- mance, static stability, dynamic stability and classical feedback control, American Institute of Aeronautics and Astronautics, Virginia, USA, (2003).
[13] M. Cook: Flight Dynamics Principles, Elsevier Ltd, Oxford, UK, second edition ed., (2007).

[14] Scholz, R and Caja, D. Box Wing Flight Dynamics in the Stage of Conceptual Aircraft Design. Hamburg University of Applied Sciences Aero - Aircraft Design and Systems Group, Deutscher Luftund Raumfahrtkongress 2012.

[15] Urso, D and Martinez-val, R. Flight Dynamics of Flying Wings. $26^{\text {th }}$ International Congress of the Aeronautical Science, ICAS 2008. 